\title{
A Digital Content Distribution Using a Group-Key and Multi-layered Structure Based on Web
}

\author{
Yun-Ji $\mathrm{Na}^{1}$ and Il Seok $\mathrm{Ko}^{2}$ \\ ${ }^{1}$ Department of Internet Software, Honam University 59-1, Seobong-Dong, \\ Gwangsan-Gu, Gwangju 506-741, South Korea \\ yjna@honam.ac.kr \\ ${ }^{2}$ School of Computer and Industrial Engineering, Yonsei University 134, \\ Shinchon-Dong, Seodaemun-Gu, Seoul 120-749, South Korea \\ isko@ctech.ac.kr
}

\begin{abstract}
Regarding the design of a multimedia digital content distribution system, the important issues are to supply a large amount of multimedia digital content to users and to guarantee the security of digital content. In this study we proposed designing a security technique for each group in a multilayered structure, and on a caching technique, which is based on this security technique, and to improve the user's response speed. Using these techniques guarantees the security of digital content distribution.
\end{abstract}

Keywords: web based system, multimedia digital content distribution, multilayered structure.

\section{Introduction}

Web based services have been activated due to the increase in network speed. Also, there is no exception in the field of digital content, in which distribution of digital content has rapidly increased [1,2]. However, almost all Web services throughout the Web have security problems due to their specific media characteristics in the Web itself. Due to this security problem, studies on security techniques have been increasingly stressed. Studies on the security techniques based on the Web consists of implementing a type of basic security technique itself [3,4], and of application techniques for the application of Web services $[2,5,6]$.

Recent studies on the transmission of digital content have been focused on the guarantee of safety and effective distribution. However, the improvement of transmission delay is also considered with this safety guarantee in the transmission of multimedia digital content. Thus, the major issue in the design of a multimedia digital content through the Web can be defined as a guarantee of the security of digital content, and fast supplement of a large amount of multimedia digital content to the user.

A content acceleration technique used in the Web is a type of user response time (web browser response time) and network traffic saving technique. In order to perform this content acceleration, a web caching method is used [7,8,9]. A web caching method increases the efficiency of fast response and network use by saving web objects, which are required by the user, who is geographically located at a close position to the Internet. Studies on the CDN (Content Delivery Network) have been increasingly 
stressed to effectively distribute digital content in the Web, in which an application of the caching technique can increase the system efficiency in a system design process. It is necessary to design a system, which reflects the characteristics of multimedia digital content, in order to increase the performance of content acceleration using a caching technique in the transmission of multimedia digital content.

In this study we proposed designing a security technique for each group in a multilayered structure, and on a caching technique, which is based on this security technique, and to improve the user's response speed. Using these techniques guarantees the security of digital content distribution.

\section{System Design}

\subsection{System Structure}

Fig. 1 presents a conceptual configuration of the system. The DCP (Digital Content Provider) is a supplier of DC (Digital Content). The DCUG (Digital Content User Group) is a user group, which is supplied by DC. Almost all users of multimedia are only interested in a certain passive action. However, a delicate encryption algorithm and certification requires a certain complicated process. This process is the cause of time delay. Thus, it is necessary to consider the transmission of DC from the view points of safety and execution speed.

Because the user of a DCUG, which is a user group of DC, applied in the proposed system can be certified in the DCUG, the user certification becomes fast and easy. In addition, an effect of the Internet traffic of DC in the proposed system decreases, and the execution speed increases due to the fact that the system will be directly affected by the DCUG cache.

Fig. 2 shows the configuration of a DCUG. A DCUG is managed by grouping it in two different groups. The first group is an authorized user group, which has the authority to use encrypted DC, and the second group is a user group, which has no authority to use encrypted DC. In addition, a DCUG uses a digital content accelerator to increase the user response speed.

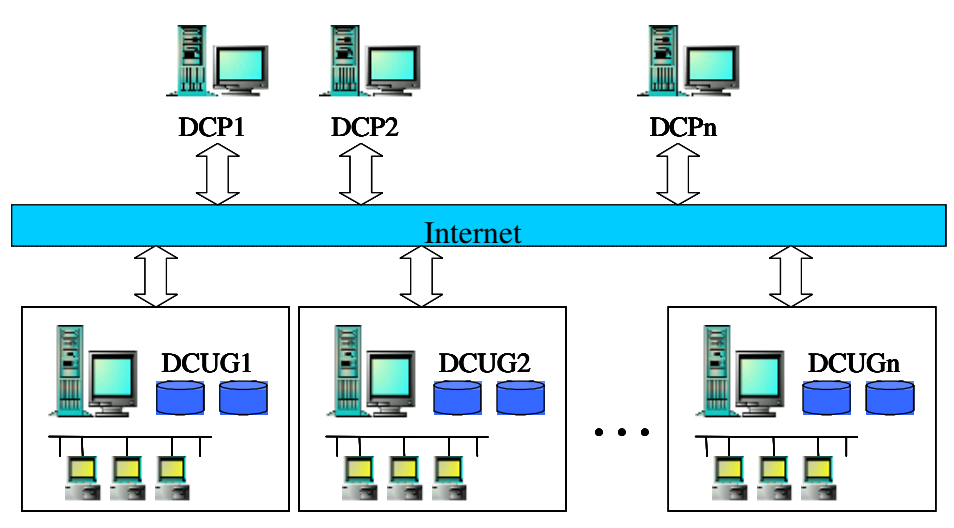

Fig. 1. System Structure 


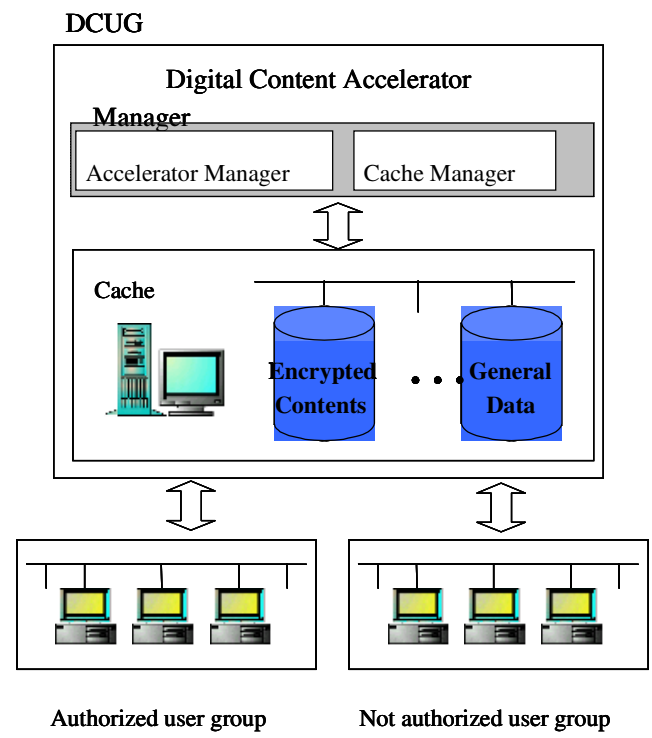

Fig. 2. DCUG Structure

A cache is managed by classifying a caching scope as an authorized user and an unauthorized user. Therefore, the structural security can be managed in the level of system by separating the DC as an authorized DC and an unauthorized DC. In the caching scope of an authorized user, the caching scope can be managed by classifying the DC as an encrypted DC and a generalized DC.

\subsection{Certification}

When the authorized user in the DCUG is unable to find the required content in the cache list, the DCUG should receive the content from the appropriate DCP server. In this case, the DCUG and DCP servers should issue a certificate by connecting the CA (Certificate Authority) before transmitting and receiving encrypted data for each other. The issuing process of the certificate is as follows.

(1) Connecting to the CA server, (2) Requesting a certificate for the CA server, (3) The CA server transmits a certificate requirement to the DCUG and DCP servers, (4) The DCP and DCUG servers produce a key pair of themselves, (5) Writing a certificate requirement, (6) The DCUG and DCP servers transmit their public keys and certificate requirements to the $\mathrm{CA}$ server, (7) The $\mathrm{CA}$ server issues a certificate including a public key by verifying the received certificate requirement, (8) The CA server saves the information of the certificate requirement of the DCUG and DCP servers and certificates to DB, (9) The CA server transmits certificates of the DCUG and DCP servers to the DCUG and DCP servers, (10) The DCUG and DCP servers save certificates received from the CA server including their private keys.

In the case of the use of the same key for all the members of the DCUG, this will cause a weakness in the security. Thus, a key agreement between the members of the 
DCUG is required. The members of the DCUG calculate the key by themselves. Table 1 presents a key agreement process between the members of the DCUG.

Table 1. Key agreement process of the members of in the DCUG

$$
\begin{aligned}
& S_{i}=i=1 u_{i} \\
& u_{i}\left(v_{i}, g^{v_{i}}=p_{i}\right) \quad u_{i+1}\left(v_{i+1}, g^{v_{i+1}}=p_{i+1}\right) \\
& x_{R} z_{q}, y=p_{i+1}^{x} \quad \stackrel{y, I=I_{i}}{\longrightarrow} \quad x^{\prime} \in_{R} z_{q}, y^{\prime}=p_{i}^{x^{\prime}} \\
& w^{\prime}=g^{x^{\prime}}, k_{i}=\left(y^{\prime}\right)^{v_{i+1}-1 z} \\
& w^{\prime}=\left(y^{\prime}\right)^{v_{i}^{-1}} \\
& y^{\prime}, I^{\prime}=I_{i+1}, z \quad z^{\prime}=h_{k}\left(2: y^{\prime}, y, I^{\prime}, I, w^{\prime}\right) \\
& w=g^{x}, k_{i}=\left(y^{\prime}\right)^{v_{i}^{-1}} \\
& z^{\prime}=h_{k}\left(2: y^{\prime}, y, I^{\prime}, I, w^{\prime}\right) \\
& z=h_{k}\left(1: y, y^{\prime}, I, I^{\prime}, w\right)
\end{aligned}
$$

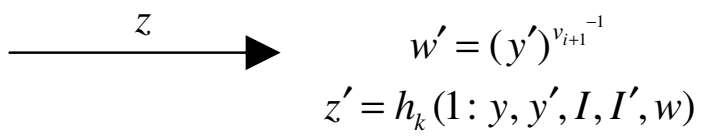

Where, the symbols noted in Table 1 are as follows.

- $u$ : members of the group communication consisted of the DCUG

- $k_{i}$ : pre-shared key through the key agreement process

- $w_{i}$ : public information calculated by the input value using a single direction function for the pre-key

- $K_{i}$ : shared DCUG keys between the members for each DCUG

- $g^{X K_{i}}$ : shared keys produced by the calculation process for each member and group manager

- $t$ : number of members of the DCUG

In addition, the initial configuration is as follows.

(1) $p: 1024$ bits prime number

(2) $q: 160$ bits of a prime factor of $p-1$

(3) $g:$ is an element of $Z_{p}^{*}$

The calculation of the modular exponent for the generator of $g$ is performed in the modulo $p$, in which $h$ is a hash function, and satisfies $h:\{0,1\}^{*} \rightarrow\{0,1\}^{q}$. In 
addition, the member of $u_{i}, u_{i+1}$ is a key agreement process, and configures a certificate and pre-shared key. The member of $u_{i}$ generates a public key of $p_{i}$ using a private key of $v_{i}$, and the member of $u_{i+1}$ also generates a public key of $p_{i+1}$ using a private key of $v_{i+1}$. Each member calculates $y, y^{\prime}$ using their opponent's public keys. This can be used to calculate a pre-shared key of $k_{i}=\alpha^{x x^{\prime}}$.

Finally, the confirmation for the pre-shared key can be performed by transmitting the value of a single direction function, which is produced by a pre-shared key. Thus, the members not only share the pre-shared key safely, but also form reliance between members.

Each member calculates the public information of $w_{i}$ using the two shared keys of $k_{i}, k_{i-1}$ of the DCUG, in which each member calculates their keys using this public information. The calculation process can be noted as follows.

(1) The member of $u_{i-1}, u_{i}$ generates a pre-shared key using a key agreement process.

(2) The member of $u_{i}$ calculates the public information of $w_{i}=h\left(k_{i}\right)-h\left(k_{i-1}\right)$ using a pre-shared key.

$h\left(k_{i}\right)$ : This applies $n$ times of single direction functions using the input value of a pre-shared key.

(3) The member of $u_{i}$ produces a small group key of $K$ for the members of a small group, which is authorized by applying an inductive method as follows, using the public information.

$u_{i}$ has the element of $, k_{i-1}, k_{i}$, and the small group key of is configured by the equation as follows.

$$
K=h\left(k_{t}\right)+h\left(k_{k-1}\right)+\cdots+h\left(k_{1}\right) \quad(\text { where, } 1 \leq l \leq n)
$$

Because $u_{i}$ recognizes the value of $h\left(k_{i-1}\right), h\left(k_{i}\right)$, the public information of $u_{i+1}$ can be calculated using the equation of $w_{i+1}=h\left(k_{i}\right)-h\left(k_{i+1}\right)$.

Because $u_{i}$ recognizes the value of $h\left(k_{i-1}\right), h\left(k_{i}\right)$, the public information of $u_{i-1}$ can be calculated using the equation of $w_{i-1}=h\left(k_{i-2}\right)-h\left(k_{i-1}\right)$.

\subsection{Transmission and Execution of DC in the DCUG}

When an authorized user in the DCUG requests DC, the DCUG manager transmits a partially encrypted DC in the cache scope to the user. Then, the user decrypts the received DC in the user's personal browser, and executes the DC using a player installed in the personal browser. Fig. 3 presents the procedure of the transmission of content from the DCUG to the DC. 


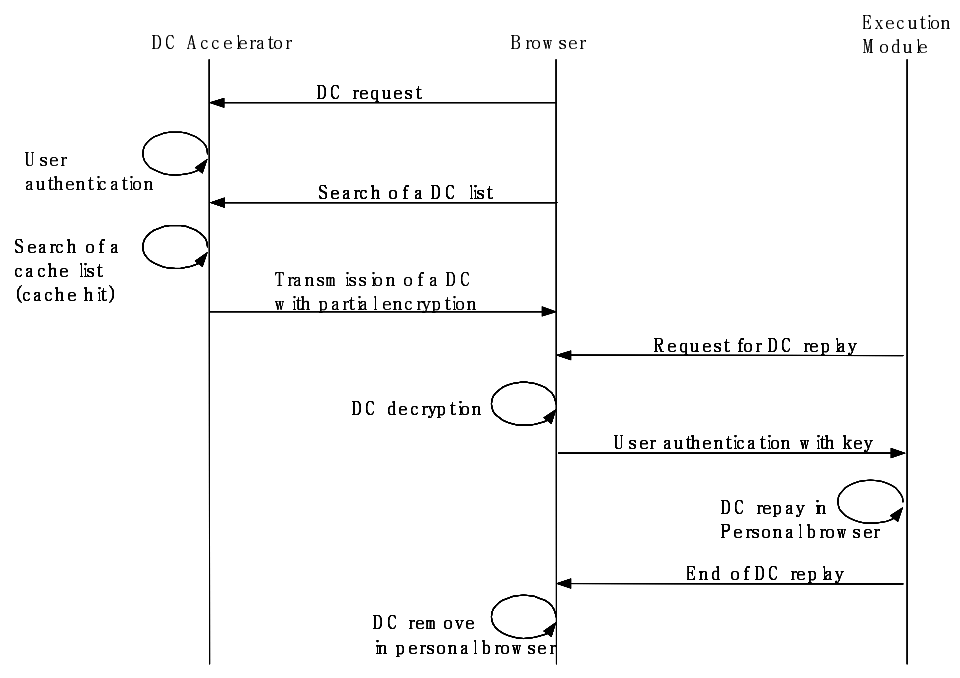

Fig. 3. Transmission and execution of DC in the DCUG

In order to execute DC in the DCUG, an exclusive browser, which has the function of opening the DC of the DCUG server, transmission of the personal information of the user, receiving DC, decryption, and play-back of DC, is required.

\section{Analysis}

Test results were compared to a frequently used existing commercial system, such as SecuMAX and Digicap, in order to verify the efficiency of the proposed system. The item of PEnc5\% and PEnc7\% present the 5\% and 7\% of partial encryption of DC, respectively. In addition, the item of Enc5\%cache 25 and PEnc5\%cache 40 present the processing speed tests of the 5\% of partial encryption of DC at the cache-hit-ratio of $25 \%$ and $40 \%$, respectively. The item of PEnc7\%cache25 and PEnc7\%cache40 present the processing speed tests of the $7 \%$ of partial encryption of DC at the cachehit-ratio of $25 \%$ and $40 \%$, respectively. Almost all commercial systems support a personal interface to assist the system security and user conveniences. As noted in Table 2, the level of security of DC was slightly reduced to improve the processing speed. This is due to the fact that the only application of encryption method and personal interface can't increase both the processing time of DC, and the level of security.

If web caching is not reflected in the system, the two existing systems present a more beneficial performance than that of the proposed system. However, the proposed system showed a high performance in the security of DC, and also presented an excellent processing speed from the aspect of considering a web caching. Because the numerical value of the test can be changed according to the test environment, it is not reasonable to conclude that the results present an absolute guideline to verify the system. However, the results revealed that the performance of the proposed system was improved compared to that of the existing commercial system. 
Table 2. Analysis of the proposed system

\begin{tabular}{|c|c|c|c|}
\hline Issue & Factors & Considerations & $\begin{array}{l}\text { Approach of the proposed } \\
\text { system }\end{array}$ \\
\hline \multirow{2}{*}{$\begin{array}{l}\text { Processing } \\
\text { Speed }\end{array}$} & $\begin{array}{l}\text { Transmission } \\
\text { speed of the } \\
\text { network }\end{array}$ & Network traffics & $\begin{array}{l}\text { Management for each DCUG } \\
\text { group/layered structure web caching }\end{array}$ \\
\hline & $\begin{array}{l}\text { User execution } \\
\text { speed }\end{array}$ & $\begin{array}{l}\text { File size of the } \\
\text { encryption/decryption }\end{array}$ & $\begin{array}{l}\text { Layered structure system/partial } \\
\text { encryption }\end{array}$ \\
\hline \multirow{2}{*}{ Security } & $\begin{array}{l}\text { Security of the } \\
\text { transmission }\end{array}$ & Safety & $\begin{array}{c}\text { Public key method/management for } \\
\text { each group }\end{array}$ \\
\hline & $\begin{array}{l}\text { Security of the } \\
\text { execution }\end{array}$ & $\begin{array}{l}\text { Speed lowering/ } \\
\text { Reducing the execution } \\
\text { process }\end{array}$ & $\begin{array}{l}\text { Security of the DCUG } \\
\text { /Certification for each DCUG group }\end{array}$ \\
\hline
\end{tabular}

The factors, which affect the processing speed of a digital content distribution system, are the delay according to the network traffic, and decryption process in user interfaces. The file size of the original sentence of DC increased due to the encryption. In addition, the encrypted transmission of a large amount of multimedia digital content, such as MP3, significantly increases the network traffic. The proposed system improves the processing speed by reducing these delay factors. The encrypted content in the DC server using a public key will be transmitted to the DCUG. The received DC can be decrypted using a personal key, and stored in a cache by applying a partial encryption. Finally, the authorized user of the DCUG will be supplied by DC, which is stored in a cache. Therefore, the traffic on the Internet for the user decreases, and the user will be affected by DC of the DCUG. In addition, because the user interface decrypts a partially encrypted content, the delay time to execute the content decreased.

The proposed system is secure, due to the fact that the DCUG, which has a personal key, can only decrypt the received DC. Because the user in the DCUG should be certified for each group, safety is guaranteed in the DCUG. In addition, the proposed system is secure enough to safely execute contents. The security of the DCUG can be guaranteed by the system itself. The authorized user of the DCUG, who is certified through the user certification, can only be allowed to access the cache list. It is necessary to make decryption when a user interface executes DC, and a certain additional security is guaranteed due to the fact that a single user, who has a proper key, can decrypt the DC.

The test results showed that the processing speed at the cache-hit-ratio of $25 \%$ was similar to that of the commercial system, and the processing speed was improved by $10 \%-18 \%$ at the cache-hit-ratio of $40 \%$. Almost all commercial web caches present over $40 \%$ of the cache-hit-ratio. Thus, the test results revealed that the performance of the proposed system improved compared to the existing commercial system. In addition, it is possible to guarantee the security of DC without any decrease in the processing time. 


\section{Conclusions}

This study designed a digital content distribution system, which can increase the execution speed, while guaranteeing the safety of DC. The proposed system introduced in this study reduces the delay factor, which is due to the network traffic during the execution of DC, using a layered web caching. In addition, this system uses a layered encryption/decryption to improve the level of security of DC. The test applied in this study compares the execution speed and level of security of the proposed system with the existing commercial system. As a result, an improvement in the level of security and execution speed of the proposed system was verified.

\section{References}

[1] Spctral Lines, "Talking About Digital Copyright," IEEE Spectrum, vol.38 Issue;6, pp.9, June 2001.

[2] Thorwkrth N. J., Horvatic P., Weis R., Jian zhap, "Security methods for MP3 music delivery," Signals, Systems and Computers, 2000. Conference Record of the Thirty-Fourth Asilomar Conference on, vol.2, pp.1831-1835. 2000.

[3] R. Rivest, A. Shamir and L. Adelman, "A Method for Obtaining Digital Signatures and Public Key Cryptosystems," Communications of the ACM, vol.21, No.2, 1978, pp.120-126

[4] Korea Information Security Agency, A Development and Analysis Report on 12bits Block Encryption Algorithm(SEED), 1998.

[5] Secumax: DRM Solution,(http://www.secumax.com)

[6] M. Just, S. Vaudenay, "Authenticated Multi-Part Key Agreement", In Advances in Cryptology - ASIACRYPT'96 LNCS 1163, pp.36-49, 1996.

[7] H. Bahn, S. Noh, S. L. Min, and K. Koh, "Efficient Replacement of Nonuniform Objects in Web Caches," IEEE Computer, Vol.35, No.6, pp.65-73, June 2002.

[8] L. Rizzo, L. Vicisano, "Replacement Polices for a Proxy Cache," IEEE/ACM Trans. Networking, vol.8, no.2, pp.158-170, 2000.

[9] C. Aggarwal, J. Wolf and P. Yu, "Caching on the World Wide Web," IEEE Trans. Knowledge and Data Engineering, vol.11, no.1, pp.94-107, 1999. 